\title{
Transposition of the supra-aortic vessels before stent grafting the aortic arch and descending aorta
}

\author{
Martin Czerny, MD, ${ }^{\mathrm{a}}$ Martin Funovics, MD, ${ }^{\mathrm{b}}$ Maria Schoder, MD, ${ }^{\mathrm{b}}$ Christian Loewe, MD, \\ Johannes Lammer, MD, ${ }^{\mathrm{b}}$ Martin Grabenwöger, MD, ${ }^{\mathrm{c}}$ Jürg Schmidli, MD, ${ }^{\mathrm{a}}$ Thierry Carrel, MD, ${ }^{\mathrm{a}}$ and \\ Michael Grimm, MD ${ }^{\mathrm{d}}$
}

\begin{abstract}
Thoracic endovascular aortic repair has broadened the spectrum of treatment options for various acute and chronic thoracic aortic diseases. In clinical practice, aneurysms of the descending aorta are rarely limited to 1 segment. Thus, various surgical and endovascular options have been developed to offer treatment to those patients with more extended descending thoracic aortic disease. We have summarized the most common methods of arch rerouting, depending on the aortic involvement, emphasizing that these techniques should be used very selectively by experienced cardiovascular surgery teams. (J Thorac Cardiovasc Surg 2013;145:S91-7)
\end{abstract}

Thoracic endovascular aortic repair (TEVAR) has broadened the spectrum of treatment options for various acute and chronic aortic diseases of the aortic arch and the descending aorta, although TEVAR at the ascending aortic level remains experimental. ${ }^{1-5}$ In clinical practice, aneurysms of the descending aorta are occasionally limited to 1 segment; however, they more frequently involve several segments. Thus, various surgical and endovascular options have been developed to allow treatment of patients with more extended descending thoracic aortic disease (termed "multisegmental") that sometimes originates at the level of the aortic arch or even in the most proximal part of the ascending aorta.

Therefore, left subclavian to left carotid artery transposition has experienced a revival for a completely different indication than that for which it was initially described. However, a substantial number of patients require extensive proximalization of the landing zone to allow endografting of pathologic features located in the proximal or middle part of the aortic arch. Consequently, double transposition (left carotid to innominate artery and left subclavian to left carotid artery) followed by total arch rerouting from the ascending aorta before TEVAR was introduced years ago and is considered an established procedure. ${ }^{6,7}$ With increasing experience, it has become obvious that these

\footnotetext{
From the Department of Cardiovascular Surgery, ${ }^{a}$ Inselspital, University Hospital Berne, Berne, Switzerland; Department of Cardiovascular and Interventional Radiology, ${ }^{\mathrm{b}}$ Medical University of Vienna, Vienna, Austria; Department of Cardiovascular Surgery, ${ }^{\mathrm{c}}$ Hospital Hietzing, Vienna, Austria; and Department of Cardiac Surgery, ${ }^{\mathrm{d}}$ Medical University of Innsbruck, Innsbruck, Austria.

Disclosures: Drs Czerny, Funovics, Schoder, Loewe, Lammer, Grabenwöger, Schmidli, Carrel, and Grimm have nothing to disclose with regard to commercial support. Read at The American Association for Thoracic Surgery Aortic Symposium, New York, New York, April 26-27, 2012.

Received for publication April 12, 2012; revisions received Aug 6, 2012; accepted for publication Nov 28, 2012; available ahead of print Dec 26, 2012.

Address for reprints: Martin Czerny, MD, Department of Cardiovascular Surgery, Inselspital, University Hospital Berne, Freiburgstr, Berne 3010, Switzerland (E-mail: martin.czerny@insel.ch).

$0022-5223 / \$ 36.00$

Copyright (c) 2013 by The American Association for Thoracic Surgery

http://dx.doi.org/10.1016/j.jtcvs.2012.11.056
}

procedures also have some limitations and unpredictable threats: 1 of the most dangerous is retrograde type A dissection. $^{8}$

The most important challenge has, therefore, been to determine which patients might be at increased risk of retrograde type A dissection and which of these patients would therefore benefit from simultaneous replacement of the ascending aorta to avoid this complication. Even if the ascending aorta requires replacement, the combined surgical and endovascular procedure can be performed at normothermia without circulatory arrest. ${ }^{9}$ Our report summarizes the most common methods of transposition of the supraaortic branches followed by endovascular treatment of the aortic arch, emphasizing that these techniques should be used very selectively.

\section{INDICATIONS FOR COMBINED APPROACHES Patient Selection}

The number of patients presenting for evaluation of thoracic aortic disease involving the aortic arch has been increasing. The technical feasibility is dependent on the extent of the disease; however, the risks of conventional surgery are primarily determined by the patient's age, frailty, and comorbidities. Patients with true aortic aneurysms usually are different to those with penetrating atherosclerotic ulcers (PAU). The latter are usually less suitable for conventional surgical approaches because of the more frequent and aggressive underlying multifocal obliterative arteriopathy. Significant comorbidities play an important role in the decision-making process and frequently correlate with the primary thoracic aortic disease. Patients with aortic dissection might constitute a subgroup who less frequently have additional comorbidities, and patients with PAU a subgroup that usually has more comorbidities. We believe that patients with multisegmental thoracic aortic disease who might require 2-step conventional surgical treatment (ascending and arch first, followed by descending aortic repair) are the best candidates for combined supra-aortic transposition followed by an endovascular approach. 


\section{Abbreviations and Acronyms \\ PAU = penetrating atherosclerotic ulcer \\ TEVAR $=$ thoracic endovascular aortic repair}

\section{Preoperative Evaluation}

The preoperative evaluation should be performed using multisclice computed tomography scans (alternatively magnetic resonance angiography) to grossly exclude significant occlusive disease of the supra-aortic branches and to optimally demonstrate the anatomy of the aortoiliac bifurcation, which is mandatory for retrograde endovascular access to the aortic arch. In addition, the imaging technique should confirm that after supra-aortic transposition, an adequate proximal neck of at least $2 \mathrm{~cm}$ along the smaller curvature of the aortic arch will be available. Patients should undergo duplex scanning of the internal carotid and vertebral arteries to exclude hemodynamically significant stenoses or occlusions. Coronary angiography is performed only in symptomatic patients and those with an extensive cardiovascular risk profile.

\section{SURGICAL APPROACH Left Subclavian-to-Left Carotid Artery Transposition}

If the lesion in the distal aortic arch involves the origin of the left subclavian artery, a direct left subclavian-to-carotid artery transposition is performed through a supraclavicular incision. Both vessels are exposed between the medial and lateral clavicular insertion of the sternocleidomastoid muscle. Selective encircling of the left vertebral artery can help to expose the central portion of the subclavian artery (Figure 1). After transection of the proximal left subclavian artery, the stump is oversewn, and an end-to-side anastomosis between the subclavian and the carotid artery is performed (Figure 2). This medial surgical approach helps to prevent damage to the recurrent laryngeal and phrenic nerves and the thoracic duct. Nevertheless, careful use of electrocautery and ligation of all lymphatic vessels are recommended.

\section{Double Transposition}

If the extent of the aortic arch lesion involves the origin of the left common carotid artery, an autologous procedure to maintain cerebral perfusion can be performed. This approach was developed in $2002 .^{10}$ In the first 2 patients, a median sternotomy approach was used and the pericardium opened. The skin incision was extended parallel to the left clavicle to offer sufficient access to the left subclavian artery. Later, the procedure was performed through a cranial hemisternotomy. Surgical access and intraoperative field are shown in Figure 3. For this procedure, it is important to provide adequate exposure of the supra-aortic vessels up to their extrathoracic level to allow tension-free anastomoses. If this is not possible, a $8-\mathrm{mm}$ Dacron interposition graft is used.

After systemic heparinization with $80 \mathrm{IU} / \mathrm{kg}$ body weight, the left common carotid artery is prepared and clamped. The vessel is transected and the proximal stump oversewn with 4-0 Prolene running suture (Ethicon, Inc, Somerville, NJ). Thereafter, the innominate artery is clamped tangentially, a longitudinal incision is made, and a side-to-end anastomosis is then performed. Arterial pressure is assessed by either the right radial artery or both radial arteries. The advantage of using the right radial artery is that any significant change in blood pressure during clamping of the innominate artery can easily be detected and corrected, with more appropriate positioning of the tangential clamp. Careful flushing and de-airing are performed before antegrade blood flow is restored to the cerebral circulation. An analogous procedure is then performed between the left subclavian artery and the already transposed left common carotid artery (Figure 4). The main advantages of this approach are that it is less invasive and the avoidance of prosthetic material for the transposition of the arch vessels, eliminating the thrombogenic risk of vascular prostheses. Nevertheless, several investigators have described excellent results using prosthetic material in such cases. ${ }^{11,12}$

\section{Total Arch Rerouting}

If the extent of the aortic arch lesion involves the origin of the innominate artery, using autologous vessels only will not be compatible with ensuring sufficient length for an adequate proximal landing zone. Thus, prosthetic material will be necessary to re-establish the cerebral and upper extremity perfusion. In these patients, we use a reversed aortobifemoral prosthesis. The procedure is performed through a median sternotomy. The pericardium is opened. After systemic heparinization with $80 \mathrm{IU} / \mathrm{kg}$, the ascending aorta is clamped tangentially. Next, an end-to-side anastomosis is performed between the aortic side of the bifurcated prosthesis and the ascending aorta, using 4-0 Prolene running suture. Optionally, a xenopericardial or Teflon felt strip can be used to reinforce the anastomosis. Thereafter, the innominate artery is transected, the proximal stump is oversewn, and an end-to-end anastomosis between 1 distal branch of the prosthesis and the innominate artery is performed with 5-0 Prolene running suture. Cerebral monitoring during this step of the operation is mandatory and is performed with an intra-arterial catheter in the right radial artery and/or using cerebral oximetry. The branch of the prosthesis is positioned ventral to the innominate vein. The blood flow is restored after flushing and de-airing. Thereafter, the second branch is placed either in front of, 


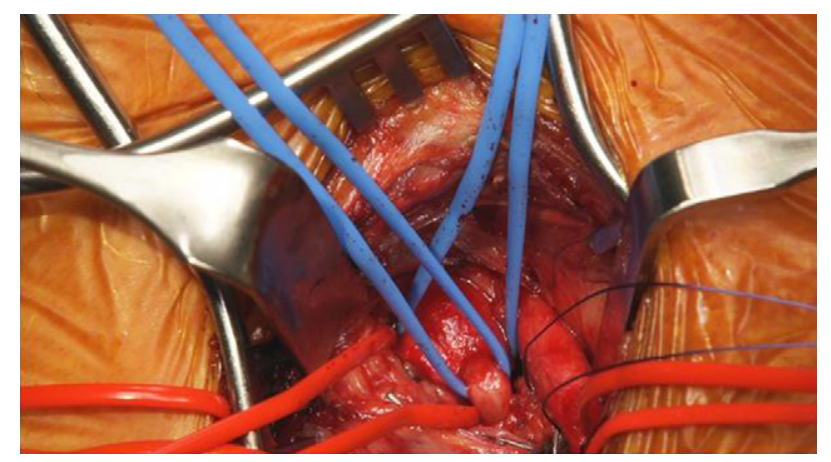

FIGURE 1. Intraoperative field before subclavian-to-carotid transposition

or behind, the innominate vein, according to the individual anatomy and to avoid compression and consequent venous inflow obstruction. The left subclavian artery is clamped, transected, and oversewn. An end-to-end anastomosis is performed with the second branch of the bifurcated prosthesis with 5-0 Prolene running suture. The intraoperative field before and after transposition is shown in Figures 5 and 6. In our experience, a $14 / 7-\mathrm{mm}$ or $16 / 8-\mathrm{mm}$ bifurcated graft is adequate for optimal size matching and provides sufficient flow, irrespective of whether Dacron or polytetrafluoroethylene grafts are used. Finally, the left common carotid artery is transected and reimplanted into the prosthetic branch to the left subclavian artery.

Both options-a simultaneous or 2-step procedure (transposition and TEVAR) - are feasible. The decision on how to proceed is determined by the findings from the individual patient evaluation, the local setting (eg, the availability of a hybrid operating room), and, finally, the institutional experience with this type of procedures.

\section{Involvement of Ascending Aorta}

When the ascending aorta must be replaced because of aneurysm or extreme atherosclerosis, the right subclavian artery is approached by a subclavicular incision and cannulated for cardiopulmonary bypass either directly or through

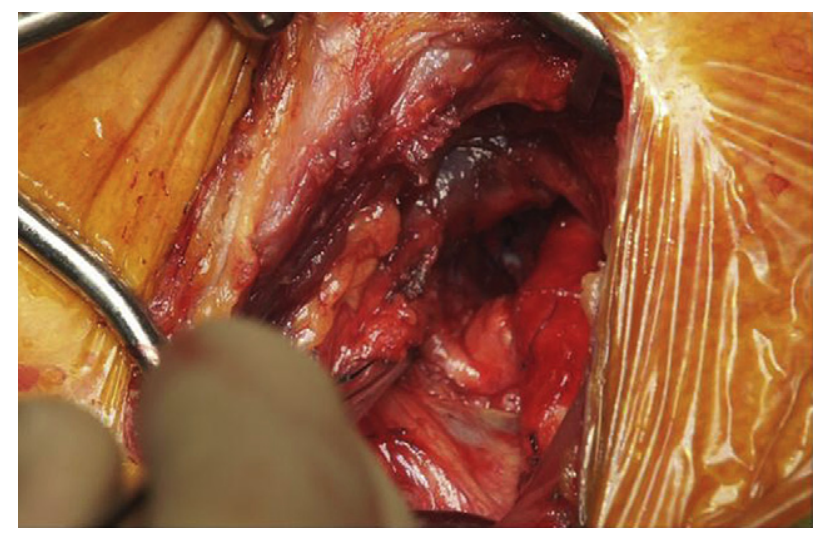

FIGURE 2. Intraoperative field after subclavian-to-carotid transposition.

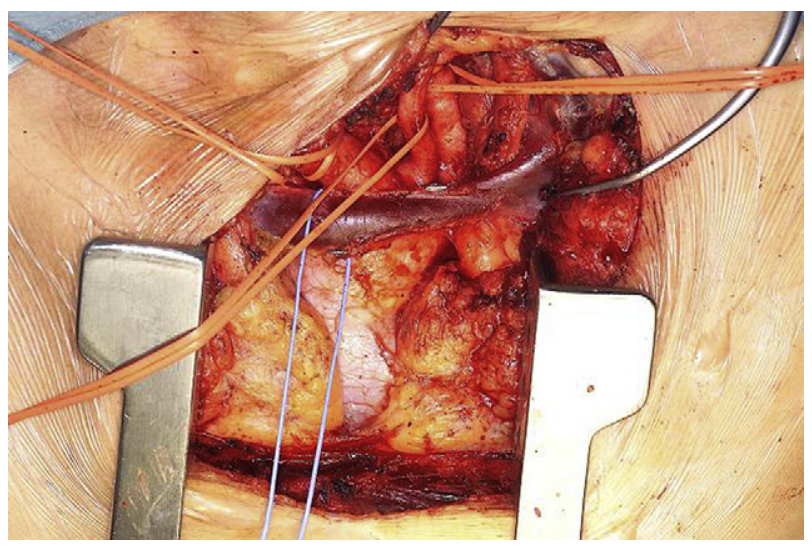

FIGURE 3. Intraoperative field before double transposition.

a side branch interposition. After median sternotomy and left cranial extension of the skin incision, the pericardium is opened, and the innominate vein and supra-aortic branches are prepared circumferentially and encircled with silastic tapes. After systemic heparinization, cardiopulmonary bypass is instituted at normothermia, and cold blood cardioplegia is used as myocardial protection. The ascending aorta is clamped just proximal to the innominate artery and resected up to the sinotubular junction. Next, the ascending aorta is replaced, and any additional procedure (coronary artery bypass grafting or aortic valve replacement) is performed. The aortic clamp is released, the aorta de-aired, and the patient is weaned from cardiopulmonary bypass. Thereafter, depending on the individual situation, a double transposition or total arch rerouting is done, as described. Figure 7 depicts replacement of the ascending aorta followed by double transposition, and Figure 8 depicts an ascending replacement followed by total arch rerouting.

\section{Stent-Graft Systems}

It was beyond the scope of the present report to describe in detail the advantages and disadvantages of all

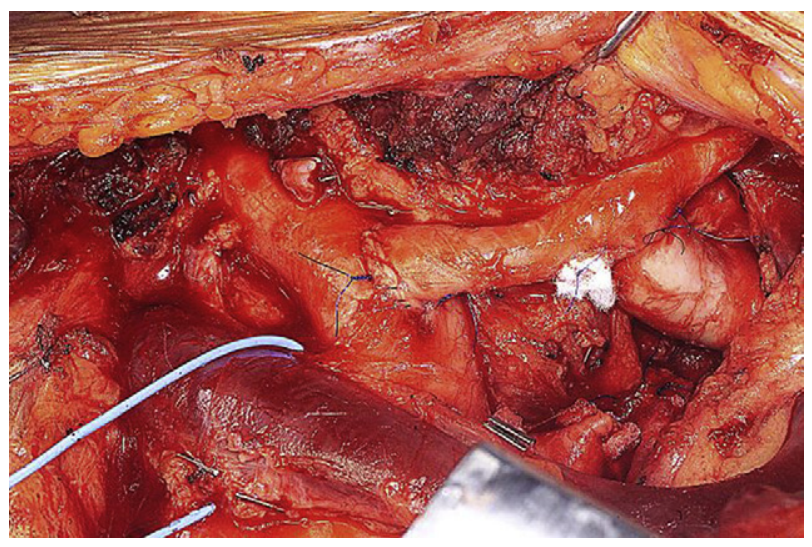

FIGURE 4. Intraoperative field after double transposition. 


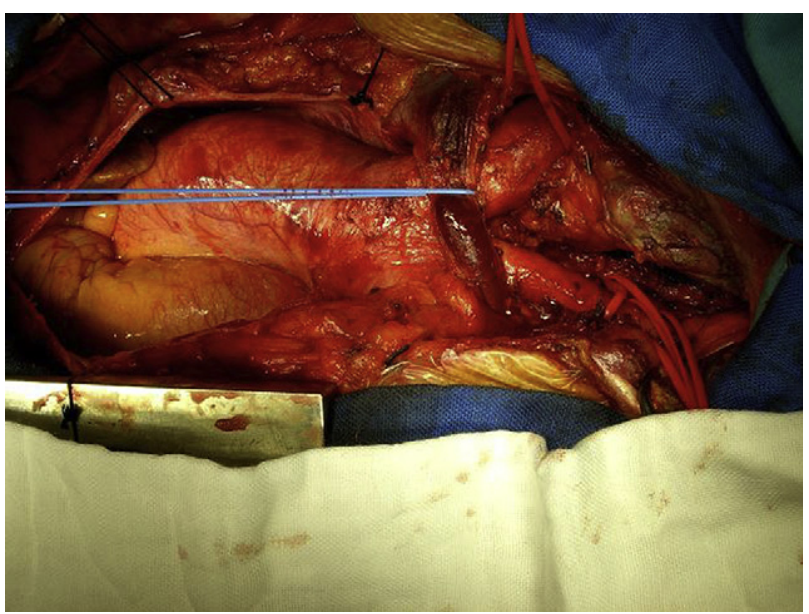

FIGURE 5. Intraoperative field before total arch rerouting.

commercially available systems. However, we would recommend the use of a device with the possibility of tip capture in the aortic arch.

\section{RESULTS \\ Surgical Procedure}

In general, the morbidity after supra-aortic reconstruction has been low. In a series of 73 supra-aortic transpositions, 1 patient sustained transient neurologic injury. ${ }^{7}$ This patient experienced multiple PAUs and had a severely calcified ascending aorta. The suspected mechanism was embolization during tangential clamping of the ascending aorta for construction of the proximal anastomosis. However, particularly after total arch rerouting, a risk of retrograde type A aortic dissection exists. The underlying mechanism of this dramatic complication remains unclear. Hypotheses include weakness of the aortic wall due to inherent disease despite a normal-size aorta; compliance mismatch between the elastic ascending aorta and the rigid stent-graft;

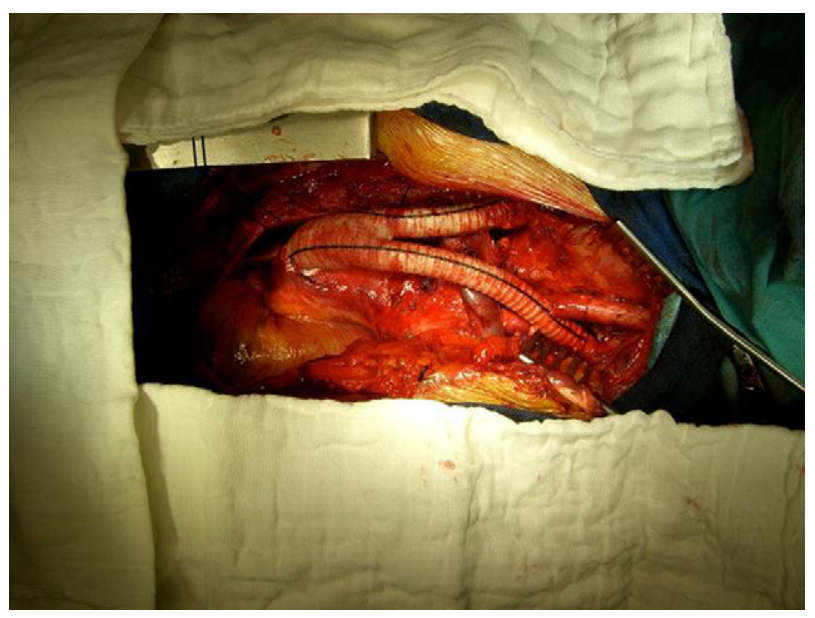

FIGURE 6. Intraoperative field after total arch rerouting.

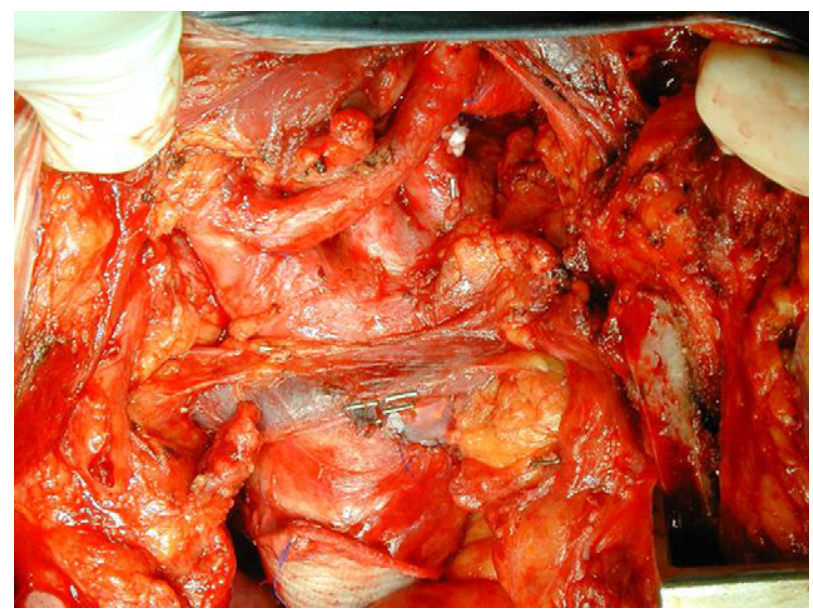

FIGURE 7. Intraoperative field after ascending aortic replacement and double transposition.

manipulation of the aorta during tangential clamping; alteration of the hemodynamics in this area; and, finally, a direct lesion resulting from proximal bare springs of some stent-grafts.

Additional complications can include injury to the left laryngeal nerve-observed in $3.6 \%$ - and injury to the thoracic duct, resulting in chronic lymph fistula or chylothorax in $1.4 \%$. Neurologic complications, either cerebral or spinal, are rare. This might be because of the careful preservation of any collateral blood supply to the brain and spinal cord resulting from the maintenance of the antegrade perfusion of the left subclavian artery. ${ }^{13}$

\section{Stent-Graft Placement}

When a few simple principles are followed, the risks of stent-graft implantation are also low. If a transfemoral approach is not possible because of narrowed or severely

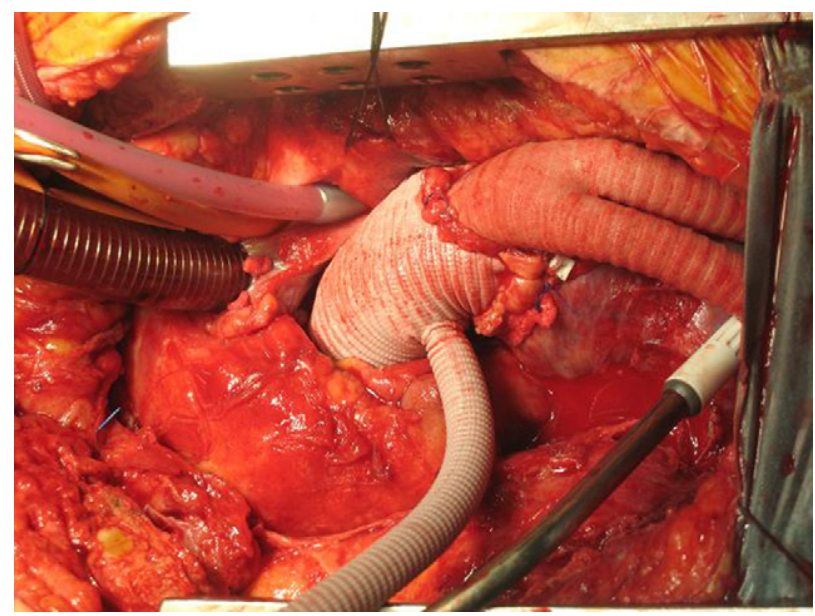

FIGURE 8. Intraoperative field after ascending aortic replacement and total arch rerouting. 
calcified vessels, the common iliac artery can be used as extraperitoneal vascular access. Usually, we recommend a 9-mm side graft to the iliac artery to facilitate stentgraft insertion. In rare cases, the infrarenal aorta or, occasionally, the ascending aorta is selected for access. Figure 9 shows a side graft to the right common iliac artery, and Figure 10 shows a side graft to an ascending aortic graft for antegrade delivery of the stent-graft.

\section{Follow-up Period and Need for Secondary Procedures}

Owing to the innovative character of these procedures and the few mid- to long-term data available, close follow-up is recommended. In our own experience, the mean follow-up period has extended up to 35 months. Other combined series have shown similar observation periods. From previous experience with stent-grafts, failures (endoleaks) due to classic mistakes such as a short landing zone, acute angulation, and limited overlap between prostheses can be expected. However, if the few basic principles described are followed, the probability of late failure and the need for any additional procedure will be very low. However, close follow-up for these patients at a dedicated center for aortic diseases for several other reasons is mandatory. These patients can develop lesions in other vascular regions that will necessitate treatment. Furthermore, the spectrum of cardiovascular risk factors observed might be responsible for any other cardiovascular disease, and clinically silent malignancies in association with smoking can be detected at a very early stage by simple chest radiography or computed tomography and might thereby be amenable to curative treatment.

\section{DISCUSSION}

Owing to the recent introduction of these procedures into the clinical routine, long-term experience is limited. Recent

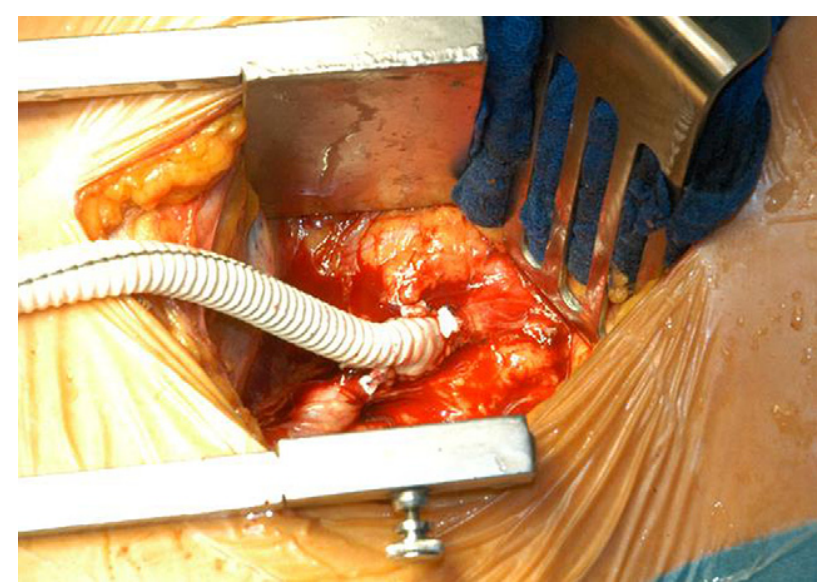

FIGURE 9. Side graft $(9 \mathrm{~mm})$ to common iliac artery for stent-graft delivery.

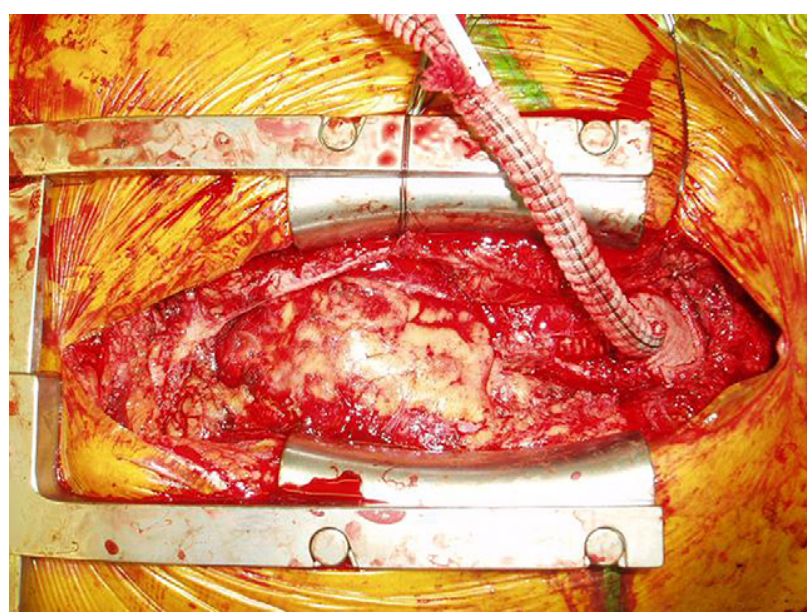

FIGURE 10. Side graft $(9 \mathrm{~mm})$ to ascending for stent-graft delivery.

work has attempted to summarize the experience in the published data currently available. ${ }^{14}$ In general, an unequivocal consensus has been reached that an anticipatory and wellselected application of combined surgical and endovascular approaches can reduce the risk for certain patient populations and enlarge the spectrum of options for elderly patients who might benefit from a less-invasive approach without extracorporeal circulation and hypothermic circulatory arrest. ${ }^{15,16}$ Some studies have tried to compare this combined approach with conventional aortic arch surgery. However, such comparisons are difficult because the patient cohorts do not match completely and the extent of disease and the clinical situation will dictate treatment on an individual basis for most patients. ${ }^{17,18}$

In our own experience, several technical aspects must be considered. Type 1 endoleak formation in the highly shearstress exposed area represented by the aortic arch must be monitored very closely. The constant friction between the stent skeleton and the graft is more pronounced within the aortic arch than within the straighter descending thoracic aorta. ${ }^{19}$ Additionally, elongation and constriction of the aorta in the longitudinal axis from functional alterations during daily life can contribute to an increase in shear stress. ${ }^{20,21}$

In our experience, both direct procedures-left subclavian-to-left carotid transposition and double transposition-can be performed safely. From a technical standpoint, total arch rerouting might be even more convenient, because the length of the native vessels is of secondary importance. Regarding left subclavian-to-left carotid transposition, it is mandatory to have adequate proximal space to safely position the clamp on the central portion of the left subclavian artery using the small extrathoracic approach. Otherwise, potential bleeding complications in this region can be controlled only with substantial effort.

In double transposition, access to the proximal portion of the supra-aortic branches is more convenient because hemisternotomy allows excellent visualization and approach. 
Large aneurysms located at the convexity of the distal aortic arch will displace the supra-aortic vessels anteriorly. This substantially facilitates the construction of the vascular anastomoses. This is not the case when a PAU occurs in a normal-size aorta or in the event of lesions involving the concavity of the aortic arch. In some instances, interposition of an 8-mm Dacron graft could be necessary to accomplish a tension-free vascular transposition. In addition, the supraclavicular extension of the incision enables mobilization of the supra-aortic branches up to an extrathoracic level, enabling tension-free vascular transposition. ${ }^{22}$

The end-to-side anastomosis between the innominate artery and left common carotid artery can be easily performed. However, circumferential dissection of the left subclavian artery and the anastomosis between the left common carotid and left subclavian artery can present some surgical challenges. In our experience, the left subclavian artery always adheres to the aneurysmal wall, and inflammation resulting from the mechanical pressure arising from the aneurysm makes it adherent to the aorta. Therefore, careful dissection is mandatory to avoid damage to the aneurysm. Finally, diameter mismatch between the left common carotid and left subclavian arteries can be present, and this should be considered when performing the left common carotid arteriotomy.

Despite the reproducibility and safety of these newer approaches, some risks remain. Any manipulation on the ascending aorta and supra-aortic branches can cause cerebral injury from embolization of atherosclerotic debris. Therefore, a no-touch (or minimal touch) technique is recommended whenever feasible. Imprecise rerouting of either the left common carotid artery or left subclavian artery can result in kinking or compression of the vessel. Brisk manipulation of the stent-graft introducer within the aortic arch can lead to detachment of soft plaques or parietal thrombotic material with consequent cerebral or peripheral embolization. Incorrect estimation of the proximal neck length can lead to unstable proximal fixation of the stent-graft, with early type 1a endoleak formation. Therefore, it is essential to determine the length of the proximal landing zone along the small curvature (concavity) of the aortic arch. Finally, retrograde type A dissection can be precipitated by the procedure itself or any manipulation. ${ }^{8,23}$ Thus, in patients with a diameter of the ascending aorta larger than $40 \mathrm{~mm}$, we recommend concomitant replacement of the ascending aorta. ${ }^{24} \mathrm{With}$ increasing knowledge of the morphology and anatomy of the ascending aorta, it is possible that more liberal replacement of the ascending aortic will be performed, together with supra-aortic transposition in the future to prevent any early and late complications in the ascending aorta.

\section{CONCLUSIONS}

A combined surgical and endovascular approach to treat multisegmental thoracic aortic aneurysmal disease can be considered an established procedure. The selection of the optimal surgical approach must be decided by a dedicated team able to provide all treatment options available and to treat all complications that might be encountered. This type of innovative approach constitutes an added value to the armamentarium of the cardiovascular surgeon in the treatment of complex and challenging pathologic features of the aortic arch.

\section{References}

1. Czerny M, Funovics M, Sodeck G, Dumfarth J, Schoder M, Juraszek A, et al. Long-term results of thoracic endovascular aortic repair in atherosclerotic aneurysms involving the descending aorta. J Thorac Cardiovasc Surg. 2010;140(6 suppl):S179-84

2. Schoder M, Czerny M, Cejna M, Rand T, Stadler A, Sodeck GH, et al. Endovascular repair of acute type B aortic dissection: long-term follow-up of true and false lumen diameter changes. Ann Thorac Surg. 2007;83:1059-66.

3. Stampfl P, Greitbauer M, Zimpfer D, Fleck T, Schoder M, Lammer J, et al. Midterm results of conservative, conventional and endovascular treatment for acute traumatic aortic lesions. Eur J Vasc Endovasc Surg. 2006;31:475-80.

4. Czerny M, Funovics M, Sodeck G, Dumfarth J, Schoder M, Juraszek A, et al. Results after thoracic endovascular aortic repair in penetrating atherosclerotic ulcers. Ann Thorac Surg. 2011;92:562-6.

5. Grimm M, Loewe C, Gottardi R, Funovics M, Zimpfer D, Rodler S, et al. Novel insights into the mechanisms and treatment of intramural hematoma affecting the entire thoracic aorta. Ann Thorac Surg. 2008;86:453-6.

6. Czerny M, Fleck T, Zimpfer D, Gottardi R, Sandner D, Cejna M, et al. Combined repair of an aortic arch aneurysm by sequential transposition of the supraaortic branches and consecutive endovascular stent-graft placement. J Thorac Cardiovasc Surg. 2003;126:930-2.

7. Gottardi R, Funovics M, Eggers N, Hirner A, Dorfmeister M, Holfeld J, et al. Supra-aortic transposition for combined vascular and endovascular repair of aortic arch pathology. Ann Thorac Surg. 2008;86:1524-9.

8. Eggebrecht H, Thompson M, Rousseau H, Czerny M, Lönn L, Mehta RH, et al. European Registry on Endovascular Aortic Repair Complications. Retrograde ascending aortic dissection during or after thoracic aortic stent graft placement: insight from the European registry on endovascular aortic repair complications. Circulation. 2009;120(11 suppl):S276-81.

9. Czerny M, Weigang E, Sodeck G, Schmidli J, Antona C, Gelpi G, et al. Targeting landing zone 0 by total arch rerouting and TEVAR - mid-term results of a transcontinental registry. Ann Thorac Surg. 2012;94:84-9.

10. Czerny M, Fleck T, Zimpfer D, Kilo J, Sandner D, Cejna M, et al. Combined repair of an aortic arch aneurysm by sequential transposition of the supraaortic branches and endovascular stent-graft placement. J Thorac Cardiovasc Surg. 2003;126:916-8.

11. Weigang E, Parker J, Czerny M, Peivandi AA, Dorweiler B, Beyersdorf F, et al. Endovascular aortic arch repair after aortic arch de-branching. Ann Thorac Surg. 2009;87:603-7.

12. Schumacher H, Von Tengg-Kobligk H, Ostovic M, Henninger V, Ockert S, Böckler D, et al. Hybrid aortic procedures for endoluminal arch replacement in thoracic aneurysms and type B dissections. J Cardiovasc Surg. 2006;47:509-17.

13. Czerny M, Eggebrecht H, Sodeck G, Verzini F, Cao P, Maritati G, et al. Mechanisms of symptomatic spinal cord ischemia after TEVAR: insights from the European Registry of Endovascular Aortic Repair Complications (EuREC). J Endovasc Ther. 2012;19:37-43.

14. Cao P, De Rango P, Czerny M, Evangelista A, Fattori R, Nienaber C, et al. Systematic review of clinical outcomes in hybrid procedures for aortic arch dissections and other arch diseases. J Thorac Cardiovasc Surg. 2012;144:1286-300.

15. Canaud L, Joyeux F, Ziza V, Branchereau P, Marty-Ané C, Alric P. Hemi-aortic arch debranching for hybrid aortic arch repair by sequential transposition of the left common carotid and subclavian arteries. J Thorac Cardiovasc Surg. Epub 2012 Apr 3.

16. Desai ND, Pochettino A, Szeto WY, Moser GW, Moeller PJ, Sodhi N, et al. Thoracic endovascular aortic repair: evolution of therapy, patterns of use, and results in a 10-year experience. J Thorac Cardiovasc Surg. 2011;142:587-94.

17. Murashita T, Matsuda H, Domae K, Iba Y, Tanaka H, Sasaki H, et al. Less invasive surgical treatment for aortic arch aneurysms in high-risk patients: a comparative study of hybrid thoracic endovascular aortic repair and conventional total arch replacement. J Thorac Cardiovasc Surg. 2012;143:1007-13. 
18. Milewski RK, Szeto WY, Pochettino A, Moser GW, Moeller P, Bavaria JE. Have hybrid procedures replaced open aortic arch reconstruction in high-risk patients? A comparative study of elective open arch debranching with endovascular stent graft placement and conventional elective open total and distal aortic arch reconstruction. J Thorac Cardiovasc Surg. 2010;140:590-7.

19. Schwartz E, Langs G, Holfeld J, Gottardi R, Loewe C, Peloschek P, et al. Segmentation and deformation analysis of the aorta in gated CTA sequences in a MDL framework. Med Image Anal. In press.

20. Harris P, Brennan J, Martin J, Gould D, Bakran A, Gilling-Smith G, et al. Longitudinal aneurysm shrinkage following endovascular aortic aneurysm repair: a source of intermediate and late complications. J Endovasc Surg. 1999;6:11-6
21. Harris P, Buth J, Mialhe C, Myhre HO, Norgren L. The need for clinical trials of endovascular abdominal aortic aneurysm repair: the Eurostar Project. J Endovasc Surg. 1997;4:72-7.

22. Czerny M, Zimpfer D, Fleck T, Hofmann W, Schoder M, Cejna M, et al. Initial results after combined repair of aortic arch aneurysms by sequential transposition of the supra-aortic branches and consecutive endovascular stent-graft placement. Ann Thorac Surg. 2004;78:1256-60.

23. Geisbüsch P, Kotelis D, Müller-Eschner M, Hyhlik-Dürr A, Böckler D. Complications after aortic arch hybrid repair. J Vasc Surg. 2011;53:935-41.

24. Bavaria J, Milewski RK, Baker J, Moeller P, Szeto W, Pochettino A. Classic hybrid evolving approach to distal arch aneurysms: toward the zone zero solution. J Thorac Cardiovasc Surg. 2010;140(6 suppl):S77-80. 